\title{
Impact of Nonstationarities on Short Heart Rate Variability Recordings During Obstructive Sleep Apnea
}

\author{
Vlasta Bari ${ }^{1}$, Luca Faes ${ }^{2,3}$, Davide Tonon ${ }^{4}$, Beatrice De Maria ${ }^{5,6}$, Giovanni Ranuzzi ${ }^{1}$, \\ Gianluca Rossato ${ }^{4}$, Alberto Porta ${ }^{1,7}$ \\ ${ }^{1}$ Department of Cardiothoracic, Vascular Anesthesia and Intensive Care, IRCCS Policlinico San \\ Donato, San Donato Milanese, Milan, Italy \\ ${ }^{2}$ BIOtech, Department of Industrial Engineering, University of Trento, Trento, Italy \\ ${ }^{3}$ Bruno Kessler Foundation, Trento, Italy \\ ${ }^{4}$ Department of Neurology, Sacro Cuore Hospital, Negrar, Verona, Italy \\ ${ }^{5}$ IRCCS Istituti Clinici Scientifici Maugeri, Istituto di Milano, Milan, Italy \\ ${ }^{6}$ Department of Electronics Information and Bioengineering, Politecnico di Milano, Milan, Italy \\ ${ }^{7}$ Department of Biomedical Sciences for Health, University of Milan, Milan, Italy
}

\begin{abstract}
Obstructive sleep apnea (OSA) is a sleep disorder characterized by breathing pauses due to collapse of the upper airways. During OSA the autonomic modulation, as noninvasively assessed through heart period (HP) variability, is altered in a time-varying way even though time-varying properties of HP fluctuations are often disregarded by HP variability studies. We performed a time domain analysis computed over very short epochs corresponding to the sole OSA events explicitly accounting for HP variability nonstationarities. Lengthmatched epochs were extracted during OSA and quiet sleep (SLEEP) in 13 subjects suffering from OSA (11

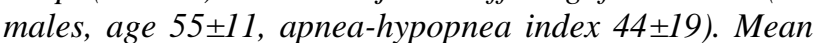
$H P$, variance and variance of the residual after exponential detrending were assessed as well as the parameters $a$ and $b$ of the exponential fitting in the form $y(n)=a \cdot \exp (b \cdot n) . H P$ mean and the parameter $a$ increased during OSA compared to SLEEP. The variance of the residual was significantly lower than original variance during both OSA and SLEEP, while the dispersion of the parameter $b$ was significantly larger. Nonstationarities were evident during both SLEEP and OSA but more dramatically apparent during OSA, thus stressing the need of accounting for them when the autonomic control during OSA is under scrutiny.
\end{abstract}

\section{Introduction}

Sleep-related breathing disorders are a frequent dysfunction, affecting about $2-4 \%$ of the adult population
[1]. Obstructive sleep apnea (OSA) has the larger prevalence, corresponding to more than $80 \%$ of the sleep disorders, and is characterized by cessations of breathing flow during sleep as a consequence of the upper airway collapse [2,3]. The event is classified as hypopnea if the collapse is partial or as apnea if it is total. OSA is known to increase the risk of mortality in association with heart failure, myocardial infarction and stroke and the risk of developing cardiovascular diseases such as hypertension. The autonomic nervous system (ANS), whose state can be noninvasively assessed by means of heart period (HP) variability analysis [4,5], is modified in correspondence of the apneic event. More specifically, sympathetic activity increases dramatically, while heart rate decreases $[6,7]$. A limitation for the study of sleep apneas is given by the fact that the HP response to an apneic event lasts up to decades of seconds, a time length remarkably shorter with respect to the usual time span covered by short-time HP variability analysis (i.e. about five minutes). Moreover, apneas introduce strong nonstationarities in the HP series. This issue is relevant because it has a deep impact over the type of HP variability analysis that can be reliably performed during apneic events [8]. Few studies analyzed apneas as a transient event, preferring to compare quasi-stationary epochs of 5 minutes close to the apnea [9], to analyze subjects during day [10] or through longer epochs as derived from $24 \mathrm{~h}$ Holter recordings [11,12]. Furthermore, the severity of the disease is usually classified according to American Academy of Sleep Medicine criteria, i.e. according to the apnea-hypopnea index (AHI), indicating the number of events occurring per hour of sleep. Evidences suggest that the apneic profile can differ from 
subject to subject, with very different average duration and different distribution of the events throughout the night in terms of type (i.e. apneas and hypopneas) and duration. Moreover, the inter-event interval might vary importantly and be quite short, thus suggesting that even in absence of an apneic event a piece of HP variability cannot be considered fully stationary in subjects with OSA. We hypothesized that a very short, time domain, HP variability analysis accounting for nonstationarities evoked by the stimulus (i.e. the upper airway collapse) could give important information about ANS state during apnea and might provide also complementary information to the AHI.

Therefore, the aim of this study is to propose a very short, time-domain, HP variability analysis in which epochs corresponding to the apneic event in OSA patients were considered. Exponential detrending was applied to locally follow nonstationarities and the residual after detrending was analyzed to characterize the HP dynamics about the trend. Results were compared to lengthmatched HP variability epochs randomly selected during quiet sleep in the same subject.

\section{Experimental protocol and methods}

\subsection{Experimental protocol and series extraction}

Thirteen subjects with OSA were enrolled (11 males, age $55 \pm 11$ yeas, mean \pm standard deviation, min-max age range: $35-71$ years; AHI $44 \pm 19$ ). The study adhered to the principles of the declaration of Helsinki for studies involving human subjects and was approved by the Ethics Committee of the Sacro Cuore Hospital, Negrar, Italy. All subjects gave an informed written consent. Subjects underwent an overnight polysomnographic recording. The surface electrocardiogram was acquired together with respiratory traces extracted at the level of the abdomen and the thorax through two respiratory inductance belts and through a nasal fluximeter and a thermistor. Oxygen saturation was measured at the finger level through a pulse oximeter. Leg movements were recorded through an electromyographic trace. Apneas/hypopneas were manually scored according to the American Academy of Sleep Medicine Criteria by a trained technician. Occurrence and classification was reviewed by a trained neurologist. AHI was computed over both apneas and hypopneas for each subject, but only OSA epochs were considered for the subsequent analysis. An hypnogram, differentiating exclusively wake and sleep periods, was utilized to assure that analysis was carried out during sleep.

From the surface electrocardiogram, HP was extracted on a beat-to-beat basis as the time distance between two consecutive R-wave peaks. HP series were checked and eventual ectopic beats or misdetections were corrected. HP series were segmented, according to the hypnogram and the apnea/hypopnea detection, into sequences obtained during quiet sleep (SLEEP) or OSA epochs. Leg movements and wake periods were excluded from the analysis as well as hypopneas.

\subsection{HRV indices computation}

A minimum length of 15 beats was chosen for analysis, shorter OSA or SLEEP epochs were discarded. To avoid biases due to an unbalanced epoch's length, OSA periods were compared to length-matched epochs extracted during SLEEP. For each subject, the HP segments classified as OSA or SLEEP were sorted according to their length from the shortest periods to the longest one. Then, the $i^{\text {th }}$ OSA segment was matched with the $j^{\text {th }}$ SLEEP one with $i=j$. If the $j^{\text {th }}$ SLEEP period was longer than the $i^{\text {th }}$ OSA one, one or more length-matched epochs were extracted without overlapping from the same $j^{\text {th }}$ SLEEP epoch. On the contrary, if the $j^{\text {th }}$ SLEEP segments was shorter than the $i^{\text {th }}$ OSA one, $j$ was increased till the next SLEEP segment longer than the $i^{\text {th }}$ OSA one was found. Once epochs of SLEEP and OSA were matched, both segments were discarded and procedure was repeated until it was not possible to find a length-matched correspondence between OSA and SLEEP segments. OSA epochs that could not be matched with SLEEP periods, due to their abnormal length or high occurrence compared to SLEEP periods, were discarded. Over each selected segment we computed the HP mean $(\mu)$ and HP variance $\left(\sigma^{2}\right)$. After fitting the original data via the exponential function $y(n)=a \cdot \exp (b \cdot n)$, where $n$ represents the progressive cardiac beat number, $y(n)$ was subtracted from the original series and the variance of the residual ( $\left.\sigma_{\text {RES }}^{2}\right)$ was computed. $\mu, \sigma^{2}, a, b$, and $\sigma_{\text {RES }}^{2}$ were calculated over each SLEEP-OSA matched pair and the median of the distribution of each index for each subject was taken as representative of that subject and utilized for the final statistical analysis [13].

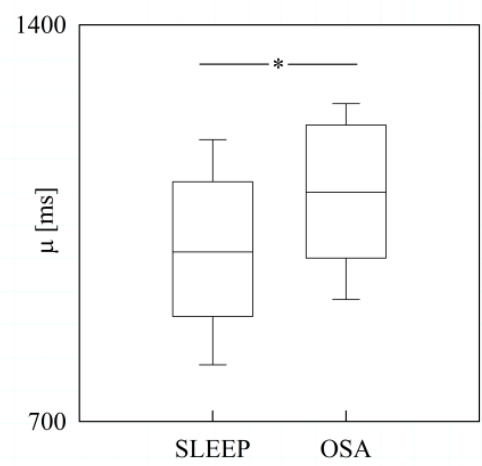

Figure 1. Box-and-whisker plot of $\mu$ during SLEEP and OSA. The symbol * indicates $p<0.05$. 


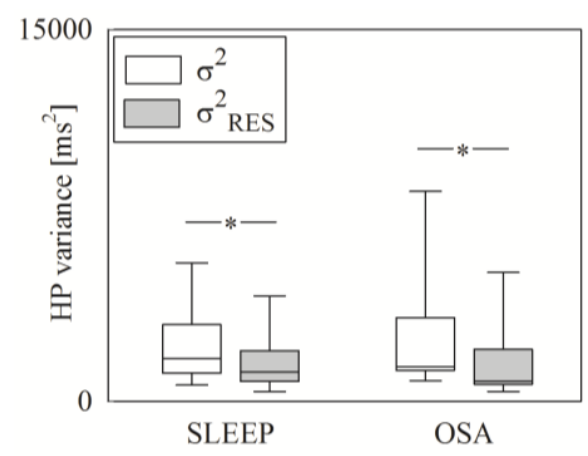

Figure 2. Grouped box-and-whisker plot of HP variance as extracted from the original series ( $\sigma^{2}$, white boxes) and after the exponential detrending ( $\sigma^{2}$ RES, grey boxes), as derived during SLEEP and OSA. The symbol * indicates $\mathrm{p}<0.05$.

\subsection{Statistical analysis}

The paired t-test, or Wilcoxon signed rank test when appropriate, was used to test the difference between SLEEP and OSA epochs in relation to the mean of parameters. Two-way repeated measures analysis of variance (Holm-Sidak test for multiple post hoc comparisons, one factor repetition) was used to assess the significance of the difference between the two conditions (i.e. SLEEP and OSA) assigned the parameter (i.e. $\sigma^{2}$ or $\sigma_{\text {RES }}^{2}$ and between the parameters assigned the condition. The interquartile range was computed to assess the dispersion of the parameters, and the F test was utilized to assess the difference between SLEEP and OSA epochs in relation to the parameter spreading. Analyses were carried out with a commercial statistical program (Sigmaplot, ver.11.0, Systat Software, San Jose, CA, USA). A $p<0.05$ was always considered as significant.

\section{Results}

Box-and-whisker plot of Fig. 1 shows $\mu$ during SLEEP and OSA epochs. $\mu$ was significantly larger during OSA than during SLEEP.

Grouped box-and-whisker plot of Fig.2 shows $\sigma^{2}$

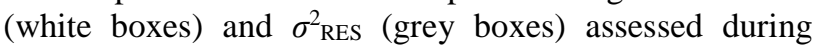
SLEEP and OSA epochs. $\sigma_{\text {RES }}^{2}$ was significantly lower than $\sigma^{2}$ and this finding held regardless of the experimental condition (i.e. SLEEP or OSA). On the contrary, assigned the parameter (i.e. $\sigma^{2}$ or $\sigma_{\text {RES }}^{2}$ ) no significant difference was observed between conditions.

Box-and-whisker plots of Fig. 3 show the parameter $a$ (Fig.3A) and $b$ (Fig.3B) of the exponential fitting carried out on HP variability segments recorded during SLEEP and OSA epochs. The parameter $a$ was larger in OSA periods (Fig.3A), thus reflecting the trend observed in Fig. 1 for $\mu$. Conversely, the parameter $b$ was similar during SLEEP and OSA epochs but it exhibited a larger dispersion during OSA. Indeed, the interquartile range of $b$ was $6.27 \times 10^{-4}$ during SLEEP and $3.54 \times 10^{-3}$ during OSA with an interquartile range ratio of OSA to SLEEP equal to 5.64 .

\section{Discussion}

This work proposes a time-domain variability analysis carried out over very short sequences of a physiological variable recorded over time epochs suitable for characterizing time varying dynamics evoked by the application of a stimulus of limited duration. The proposed analysis is based on a detrending procedure and on the computation of parameters describing the trend and the dynamics after detrending (i.e. the residual). The approach suggests a procedure to compare the parameters extracted in presence of the stimulus with those derived from control periods selected from the same variability recording when the stimulus is absent. In this specific application the function utilized to fit the trend belongs to the class of the exponential functions, the stimulus is an apneic event resulting from the upper airway collapse occurring overnight, and the variability recording is the beat-to-beat HP series continuously recorded from the surface electrocardiogram.

Time domain analysis confirmed that HP mean increased during OSA compared to SLEEP [7]. Conversely, HP variance was not significantly modified during OSA, even though a trend towards an increase was detectable [14]. The original HP variance was significantly higher than that after detrending both during SLEEP and OSA periods. This finding stresses the relevance of nonstationarities present in very short HP variability segments and the necessity of accounting for their presence in both SLEEP and OSA epochs [8]. According to this finding, traditional analysis tools based on assumption of stationarity, such as e.g. time invariant spectral methods or time domain methods assuming the stability of the mean, might be inadequate to the aim at quantifying cardiac control during OSA [9-10]. Also the traditional length of the frame utilized for short-term HP variability analysis (i.e. about 5 minutes) should be revised given the speed of adaptation of HP to the occurrence of OSA and its brief duration compared to the frame length.

The exponential fitting parameters $a$ and $b$, namely the onset of the fitting curve at $n=0$ and the time constant of the exponential decay respectively, were able to differentiate SLEEP and OSA periods. In fact, $a$ was higher during OSA according to the larger values of the HP mean. Conversely, $b$ was similar in the two experimental conditions but its dispersion about the mean value was significantly larger during OSA than SLEEP. Since the analyses were performed on length-matched epochs belonging to the same subject, we can exclude that this difference could be due to the inter-subject variability or variable length of the sequences. We 

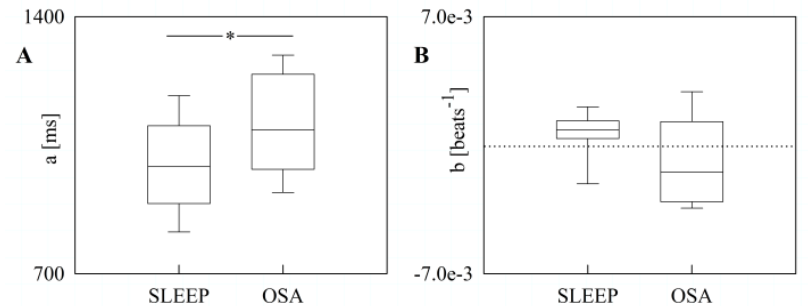

Figure 3. Box-and-whisker plot of $a$ (A) and $b$ (B) as derived from the exponential fitting of the HP series during SLEEP and OSA epochs. The symbol * indicates $p<0.05$ between means. The dispersion of $b$ during OSA was significantly greater than that during SLEEP with $p<0.05$. The dotted line in (B) indicates the zero value.

hypothesize that such larger dispersion of $b$, spanning from negative to positive time constants, is a marker of the heterogeneity of the response of the OSA group to the apneic event. This heterogeneity outlines further the relevance of the nonstationarities present in HP variability during OSA and deserves further investigation because it could provide a patient-specific characterization according to the severity of the disease.

\section{Conclusions}

This study proposes a time-domain analysis performed over very short HP variability recordings extracted during OSA. Results were contrasted with length-matched segments during SLEEP periods. Our findings stress the relevant impact of nonstationarities, especially during OSA, thus remarking the need of their description when studying cardiac autonomic control from spontaneous HP fluctuations in OSA patients. The proposed approach is general and can be applied to describe any variability series characterized by transients induced by a stressor of limited duration via the quantification of the time varying dynamic (i.e. the trend) and the fluctuations around the trend (i.e. the residual). As to the specific application to OSA patients, further studies are needed to better understand whether the proposed indices provide complementary information to more traditional scores utilized to characterize OSA patients such as AHI and might be fruitfully exploited to stratify OSA population.

\section{References}

[1] T. Young, M. Palta, J. Dempsey, J. Skatrud, S. Weber and S. Badr, "The occurrence of sleep-disordered breathing among middle-aged adults," N. Engl. J. Med., vol. 328, pp. 1230-1235, 1993.

[2] A. Malhotra and D.P. White, "Obstructive sleep apnoea," Lancet, vol. 360, pp. 237-245, 2002.

[3] M.O. Mendez, J. Corthout, S. Van Huffel, M. Matteucci, T. Penzel, S. Cerutti and A.M. Bianchi, "Automatic screening of obstructive sleep apnea from the ECG based on empirical mode decomposition and wavelet analysis," Physiol. Meas., vol. 31, pp. 273-289, 2010.

[4] N. Montano, T. Gnecchi-Ruscone, A. Porta, F. Lombardi, M. Pagani and A. Malliani, "Power spectrum analysis of heart rate variability to assess the changes in sympathovagal balance during graded orthostatic tilt," Circulation, vol. 90, pp. 1826-1831, 1994.

[5] A. Marchi, V. Bari, B. De Maria, M. Esler, E. Lambert, M. Baumert and A. Porta, "Calibrated variability of muscle sympathetic nerve activity during graded head-up tilt in humans and its link with noradrenaline data and cardiovascular rhythms," Am. J. Physiol., vol. 310, pp. R1134-R1143, 2016.

[6] V.K. Somers, M.E. Dyken, M.P. Clary and F.M. Abboud, "Sympathetic neural mechanisms in obstructive sleep apnea,” J. Clin. Invest., vol. 96, pp. 1897-1904, 1995.

[7] S. Andreas, G. Hajak, B. von Breska, E. Rüther and H. Kreuzer, "Changes in heart rate during obstructive sleep apnoea,” Eur. Respir. J., 5, 853-857. 1992.

[8] V. Magagnin, T. Bassani, V. Bari, M. Turiel, R. Maestri, G.D. Pinna and A. Porta, "Non-stationarities significantly distort short-term spectral, symbolic and entropy heart rate variability indexes," Physiol. Meas., vol. 32, pp. 1775-1786, 2011.

[9] M.J. Lado, A.J. Méndez, L. Rodríguez-Liñares, A. Otero and X.A. Vila, "Nocturnal evolution of heart rate variability indices in sleep apnea," Comput. Biol. Med., vol. 42, pp. 1179-1185, 2012

[10] K. Narkiewicz, N. Montano, C. Cogliati, P.J. van de Borne, M.E. Dyken and V.K. Somers, "Altered cardiovascular variability in obstructive sleep apnea," Circulation, vol. 98, pp. 1071-1077, 1998.

[11] A. Noda, F. Yasuma, T. Okada and M. Yokota, "Circadian rhythm of autonomic activity in patients with obstructive sleep apnea syndrome," Clin. Cardiol., vol. 21, pp. 271-276, 1998.

[12] M. Aydin, R. Altin, A. Ozeren, L. Kart, M. Bilge and M. Unalacak, "Cardiac autonomic activity in obstructive sleep apnea: time-dependent and spectral analysis of heart rate variability using 24-hour Holter electrocardiograms," Tex. Heart Inst. J., vol. 31, pp. 132-136, 2004.

[13] A. Porta, L. Faes, M. Masé, G. D’Addio, G.D. Pinna, R. Maestri, N.Montano, R. Furlan, S.Guzzetti, G. Nollo and A. Malliani, "An integrated approach based on uniform quantization for the evaluation of complexity of short-term heart period variability: Application to 24h Holter recordings in healthy and heart failure humans," Chaos, vol. 17, art. no. 015117. 2007.

[14] A. Flevari, E. Vagiakis and S. Zakynthinos, "Heart rate variability is augmented in patients with positional obstructive sleep apnea, but only supine LF/HF index correlates with its severity," Sleep Breath., vol. 19, pp. 359$367,2015$.

Address for correspondence.

Dr. Vlasta Bari, PhD

IRCCS Policlinico San Donato - Department of Cardiothoracic, Vascular Anesthesia and Intensive Care

via F. Fellini 4, 20097 San Donato Milanese, Milan, Italy

Tel: +390252774381

e-mail: vlasta.bari@grupposandonato.it 